\title{
L'orobe (Vicia ervilia L. Willd.) au Maroc
}

Histoire, nomenclature et usage d'une culture marginalisée

The bitter vetch (Vicia ervilia L. Willd.) in Morocco: history, nomenclature and use of a marginalized culture

\section{Salama El Fatehi et Mohammed Ater}

\section{OpenEdition}

\section{Journals}

\section{Édition électronique}

URL : https://journals.openedition.org/ethnoecologie/3128

DOI : 10.4000/ethnoecologie.3128

ISSN : 2267-2419

\section{Éditeur}

Laboratoire Éco-anthropologie

\section{Référence électronique}

Salama El Fatehi et Mohammed Ater, "L'orobe (Vicia ervilia L. Willd.) au Maroc », Revue d'ethnoécologie [En ligne], Supplément 1 | 2017, mis en ligne le 17 octobre 2017, consulté le 19 novembre 2021. URL : http://journals.openedition.org/ethnoecologie/3128; DOI : https://doi.org/10.4000/ethnoecologie. 3128

Ce document a été généré automatiquement le 19 novembre 2021.

\section{cc) (†)}

Revue d'ethnoécologie est mis à disposition selon les termes de la licence Creative Commons Attribution - Pas d'Utilisation Commerciale - Pas de Modification 4.0 International. 


\title{
L'orobe (Vicia ervilia L. Willd.) au
}

\section{Maroc}

\author{
Histoire, nomenclature et usage d'une culture marginalisée \\ The bitter vetch (Vicia ervilia L. Willd.) in Morocco: history, nomenclature and \\ use of a marginalized culture
}

Salama El Fatehi et Mohammed Ater

\section{Introduction}

1 L'agrobiodiversité est une composante essentielle et vitale de la réponse aux défis des changements globaux en matière de sécurité alimentaire. Cependant, on peut constater que c'est loin d'être une évidence pour les intervenants dans les chaînes de production des aliments. En effet, les sciences agronomiques et les sciences de la nutrition semblent ignorer le lien entre plantes alimentaires cultivées et biodiversité. Pourtant, l'agrobiodiversité est essentielle pour la sécurité alimentaire future et elle nécessite une approche intégrative selon quatre angles : i) ressources génétiques et écologie ; ii) gouvernance et politiques agricoles; iii) alimentation et santé et iv) changements globaux (Zimmerer \& De Hann 2017). Elle peut donc être abordée sous l'angle central de la durabilité et la soutenabilité des systèmes de production pour garantir la qualité et la sécurité alimentaire (Perreault 2005). Dans cet ordre de pensées, l'agriculture durable serait celle pratiquée à petite échelle et qui permettrait de conserver des systèmes de production résilients et durables (Gonzalez 2011).

2 Les agroécosystèmes de montagnes semblent particulièrement favorables pour la conservation de l'agrobiodiversité. En effet, Zimmerer et al. (2016) ont démontré une relation significative entre l'altitude et l'agrobiodiversité dans les montagnes des zones pré-équatoriales et équatoriales $d u$ monde en tenant compte de l'influence de l'éloignement géographique. Ainsi, parmi les $\operatorname{SIPAM}^{1}$ on compte plusieurs agroécosystèmes de montagnes en Asie et en Amérique latine. Au Maghreb, les seuls SIPAM reconnus sont les oasis. Cependant, les agroécosystèmes de montagnes au Maroc 
représentent un important réservoir de l'agrobiodiversité et un refuge pour les cultures marginales comme le montre l'exemple des montagnes du Rif et du pays Jbala (Hmimsa \& Ater 2008, Ater \& Hmimsa 2008, 2013). Ce sont des agroécosystèmes caractérisés par la pratique de l'agriculture traditionnelle basée sur la polyculture et l'utilisation des variétés locales. L'un des aspects important de l'agrobiodiversité de ces agroécosystèmes est la persistance de cultures rares et marginales. On peut citer comme exemples : le petit épeautre, le seigle et des variétés d'orge nue chez les céréales et chez les légumineuses, des vesces, des gesses et la dolique. La marginalisation de ces cultures n'est pas un processus récent mais au contraire ancien et qui résulte de plusieurs facteurs notamment des facteurs historiques comme l'introduction de nouvelles cultures (Hernandez Bermejo \& Leon 1994). Ainsi certaines cultures en provenance du nouveau monde ont marginalisé des cultures autochtones comme le maïs chez les céréales de printemps qui a remplacé les céréales autochtones (sorgho, mil et millet). Actuellement, ce processus de marginalisation s'est accéléré suite à la rupture de l'éloignement et de l'incidence des politiques incitatives à la modernisation de l'agriculture. Ces facteurs correspondent à différentes faces des effets de la mondialisation et de la globalisation de l'économie. Ce bouleversement est relativement récent sur la rive sud de la Méditerranée et spécialement les pays du Maghreb où l'intensification de la régression des pratiques agricoles traditionnelles date seulement de la deuxième moitié du siècle dernier.

Dans cette contribution nous allons traiter l'exemple d'une culture minorée, actuellement considérée comme marginale et sous-utilisée dans les agroécosystèmes traditionnels des montagnes du Rif occidental. Il s'agit d'une vesce, Vicia ervilia (L.) Willd. ${ }^{2}$ dont le nom vernaculaire arabe est « kersannah », " ers » en français et "bitter vetch» en anglais. C'est une culture très ancienne dont l'histoire remonte à la préhistoire (Paléolithique) et de ce fait elle peut être associée à l'origine de l'agriculture et à sa pratique dans la région méditerranéenne. Actuellement, il s'agit d'une culture rare de nos régions où elle est considérée plutôt comme une plante fourragère et utilisée exclusivement en alimentation animale. L'histoire de cette culture montre cependant que cela n'a pas été toujours le cas. Autrefois, c'était un légume sec utilisé également pour l'alimentation et la pharmacopée humaine sous différentes formes.

Des travaux récents (El Fatehi et al. 2014 et 2016) ont montré que les écotypes locaux de cette espèce renferment une diversité génétique et agromorphologique dont certains caractères recherchés dans la réponse aux changements climatiques comme la résistance à la sécheresse. Cette espèce est donc un bon exemple de l'intérêt de l'agrobiodiversité, d'autant plus que sur la même aire coexistent des formes sauvages avec les formes cultivées. La conservation des complexes des plantes cultivées et espèces sauvages apparentées est une priorité pour la résilience des espèces cultivées (Castañeda-Álvarez et al. 2016). D'autre part, l'intérêt pour la réhabilitation des plantes cultivées anciennes concerne également V. ervilia (Enneking \& Miller 2014).

\section{Fruit ou légume : quelle signification?}

5 Il faut noter, que si le terme "fruit » a une signification au sens botanique, le mot « légume » n'en a aucune. En effet, du point de vue botanique, le fruit est un organe issu de la reproduction chez les plantes à fleurs (Angiospermes) et correspond à la transformation de l'ovaire après fécondation. La signification botanique du « fruit » ne 
correspond donc pas à son utilisation dans la vie courante. Ainsi, certains «légumes » correspondraient plutôt à des fruits comme par exemple une tomate, un poivron, une courgette, une aubergine ou des gousses de haricots. Par contre, un légume ne correspond à aucun organe particulier chez les plantes et suivant les cas il peut correspondre à : une feuille, une tige, une racine, un bulbe, un tubercule, une fleur ou un fruit. Ainsi, la définition usuelle d'un légume est qu'il s'agit d'une plante potagère, c'est-à-dire, une plante herbacée dont une partie est utilisée dans l'alimentation humaine. Et on peut distinguer des légumes frais et des légumes secs. Les légumes secs correspondent à des "graines » et appartiennent, au moins en Méditerranée à des plantes de la famille des Fabacées. Les graines sont des organes de dissémination chez les plantes supérieures ou «Spermaphytes ». Elles sont riches en réserves nutritives et bien adaptées pour la conservation, ce qui leur confèrent un double avantage: une ressource nutritive utile pour l'alimentation et la possibilité de stockage pour les réserves alimentaires. Ce type de légumes est également associé aux féculents riches en réserves glucidiques principalement de l'amidon. Elles sont également riches en différents types d'éléments nutritifs comme les protéines et les oligoéléments. C'est pour cela que les légumes secs étaient considérés comme une source d'alimentation importante depuis tous les temps.

\section{Une culture ancestrale de la Méditerranée}

6 Vicia ervilia fait partie des premières plantes domestiquées par l'homme (Zohary et al. 2012). L'ancêtre de la plante cultivée n'est autre qu'une forme sauvage de la même espèce et qui s'en différencie par des caractères favorisés par la sélection comme la déhiscence des gousses, la taille des graines et de la plante. L'origine de cette plante correspond à l'aire de la Méditerranée orientale englobant l'Anatolie, l'Arménie, le Nord de l'Irak et le Liban. Elle a ensuite rayonné dans tout le pourtour méditerranéen par deux formes: une forme domestiquée cultivée et une forme spontanée adventice associée aux cultures.

7 La culture de Vicia ervilia est très ancienne et remonte à la préhistoire. En effet, des travaux archéobotaniques montrent sa présence dès le Moustérien, aux environs de 65 000-48 000 ans av. J-C. à Kebara Cave en Israël (Lev et al. 2005). D'autres sites ont révélé sa présence dans l'épipaléolithique, environ 13 400-11 350 ans av. J-C. à Abu Hureyra (Hillman 1975, Hillman et al. 1989 et 2001), et environs 11 800-11 300 ans av J-C. à Mureybet (Van Zeist \& Casparie 1968, Van Zeist \& Bakker Heeres 1986).

8 En Méditerranée occidentale, cette culture semble plus récente et apparait seulement au début du néolithique. Des graines de Vicia ervilia ont été découvertes en Espagne aux environs de $7365 \pm 47$ ans av. J-C. à La Vaquera et à $7098 \pm 60$ ans av. J-C. à Mármoles (Buxó 1997, Peña-Chocarro \& Zapata 2010, Pérez-Jordà et al. 2011, Zapata et al.2004). Des semences de Vicia ervilia ont été découvertes dans $15 \%$ des sites prospectés (35) dans le sud de la France à la fin de l'âge du bronze, aux alentours de 1400-1300 à 800 ans av. J-C. (Bouby 2003).

9 En ce qui concerne le Maroc, et pour faire le parallèle avec la péninsule ibérique, il faut noter que les données archéobotaniques sont plus rares et concernent un nombre réduit de sites. De ce fait nous nous baserons seulement sur les données de deux sites du nord du Maroc, Kaf Taht Alghar étudié par Ballouche et Marinval (2003) et Ifri oudadane étudié par Morales et al. (2013). Pour le premier site, les études montrent 
l'existence d'espèces domestiquées dans le Néolithique initial et confirment la présence des premières formes reconnues d'agriculture au Maroc. Ces résultats témoignent de l'ancienneté de l'agriculture dans cette région de l'Afrique du Nord. La céréaliculture est affirmée dès le $\mathrm{VI}^{\mathrm{e}}$ millénaire av. J-C. aux environs de 6500. Parmi les espèces cultivées on a retrouvé des légumineuses, mais aucune trace de Vicia ervilia n'a été observée. Pour le deuxième site, les résultats indiquent la présence de plantes domestiquées, des céréales (orge polystique, engrain ou petit épeautre et blé dur) et des légumineuses (lentille et pois) dans le néolithique. Une lentille a été datée à $7611 \pm 37$ av. $\mathrm{J}-\mathrm{C}$. représentant la plus ancienne date directe d'une graine de plante domestiquée au Maroc et en Afrique du Nord.

10 Ces données attestent à la fois de l'ancienneté de cette culture en Méditerranée occidentale dans le sud de la France et en Espagne et son absence au Maroc. Cependant, la signification de cette absence serait à relativiser étant donné la rareté des données archéobotaniques de la préhistoire et de la protohistoire au Maroc.

\section{Nomenclature et diversité des noms vernaculaires en Méditerranée occidentale}

11 Du point de vue de la nomenclature botanique les choses sont claires, il s'agit de Vicia ervilia (L.) Willd. (1802) dont le basionyme ${ }^{3}$ est Ervum ervilia L. (1753). Il existe dans la littérature deux synonymes : Ervilia sativa Link. et Lens pygmea Grossh.

Cependant, si du point de vue scientifique les choses sont claires, il en est autrement au niveau des noms vernaculaires utilisés en Méditerranée occidentale dont la diversité peut engendrer une certaine confusion. En effet, on note une grande diversité de noms utilisés dans les différentes langues de la région comme le français, l'espagnol ou le portugais. Cette grande diversité de la nomenclature dans la littérature complique parfois les recherches bibliographiques.

13 Ainsi, il existe plusieurs variantes du nom vernaculaire français dont les plus courantes sont ers, ervilier ou vesce ervilière. Elles seraient dérivées du nom latin ervum qui serait d'origine grec et signifie lentille selon Carnoy (1958). Ils existent d'autres noms comme la lentille noire, lentille bâtarde, pois mauresque, orobe, faux-orobe..... Dans cette étude nous adopterons le nom français utilisé au Maroc, l'orobe.

En espagnol castillan la diversité des noms vernaculaires est phénoménale. En effet, on ne compte pas moins de 39 noms ou déclinaisons différentes ${ }^{4}$. Le nom le plus utilisé actuellement est " yero ». Cependant, on trouve des noms comme alcarceña, alcarceña et alcarceño qui dériveraient de l'arabe "kersannah" et témoignent de l'influence arabo-musulmane. Corriente (2008) dans son dictionnaire des noms d'origines arabes et étrangères cite "alcarceña» et atteste son origine arabe. Parmi les noms cités, on trouve un autre nom représentatif de cette origine, "alcarceña de Toledo". Montemayor (1996), dans un chapitre consacré à la présentation d'un domaine agricole de son ouvrage "Tolède entre fortune et déclin (1530-1640)», cite parmi les cultures annuelles importantes de l'époque le froment (candeal), l'orge, le seigle et l'ers (alcarceña) en soulignant que cette exploitation a gardé la même allure qu'au XIV et Xve siècles. 
15 En portugais ${ }^{5}$, on note également une grande diversité des noms vernaculaires avec au moins 13 noms connus. Cependant, contrairement à l'espagnol, aucun des noms portugais ne semble avoir une origine arabe. Par contre, Barkai (1998) a relevé l'existence du nom « kersannah » en soulignant son origine arabe dans des traités de médecine en hébreux du moyen âge en Europe méditerranéenne.

17 L'étymologie du nom vernaculaire en espagnol de Vicia ervilia montre l'importance de l'influence arabe sur la pratique de cette culture en Espagne et pourrait par conséquent laisser penser que cette culture a été introduite par les arabes. Or les données de l'archéobotanique montrent clairement qu'il s'agirait plutôt d'une culture ancienne en Espagne et antérieure à la présence arabe.

18 En ce qui concerne le nom vernaculaire en arabe, au Maroc, on connaît un seul nom qui est karssena, kerssena ou kerssanah ${ }^{6}$ suivant les sources, dont la transcription phonétique peut être kərsənna ou kərsānna (ILDIS World Database of Legumes 2010). Cette plante étant actuellement cultivée dans des zones peuplées par une population arabophone nous n'avons relevé aucun nom berbère. Pourtant, nous avons relevé une confusion dans certains travaux réalisés en zone berbérophone dans l'Atlas et qui citent comme traduction de l'ers le nom berbère de kiker ou ikiker qui a une origine latine "cicera " qui signifie pois chiches (CBTHA 2002). Nous pensons qu'il s'agit d'une erreur, car à notre avis ikiker désigne plutôt une gesse encore cultivée dans la région d'Azilal (Lathyrus cicera) ou une autre vesce dont la culture a disparu dans ces régions (Vicia sativa).

Le nom vernaculaire français utilisé par la littérature agronomique au Maroc pose également un problème. En effet, contrairement au nom vernaculaire le plus connu en France (?) qui est « ers », on remarque plutôt l'utilisation du nom "orobe ». Foury (1954), explique l'origine de ce choix par une confusion des responsables des statistiques de l'administration du protectorat ${ }^{7}$ qui l'ont identifié par erreur comme étant Orobus hirsutus L. Les services de la recherche agronomique au Maroc continuent encore à utiliser " orobe " pour désigner Vicia ervilia (Bounejmate 1997). Il faut noter que Leclerc (1874) en Algérie a aussi utilisé « orobe » dans la traduction de kersannah à partir de manuscrits arabes anciens. Dans la zone sous protectorat espagnol, le nom espagnol utilisé dans les documents agronomiques et dans les statistiques est " yero ».

\section{Typologie des usages et utilisations}

Actuellement, l'utilisation principale des graines de l'orobe dans l'ensemble de la Méditerranée se limite à l'alimentation animale chez les ruminants, notamment les bovins (Enneking 1994). Elle peut aussi être utilisée comme plante fourragère, verte ou sèche (Miège 1938, Villax 1963). Cependant, au cours de son histoire, l'orobe a servi à d'autres usages comme légume sec dans l'alimentation humaine ou dans la pharmacopée pendant certaines périodes. En comparaison avec les autres légumineuses elle a été toujours peu appréciée et utilisée seulement quand les gens étaient forcés de la consommer spécialement dans les périodes de famine et de disette. L'impopularité de l'orobe trouve son explication dans sa faible appétence. En effet, la graine de l'orobe présente deux inconvénients majeurs pour l'alimentation humaine : un goût amer et la présence d'une substance toxique, la canavanine (Enneking 1995). Pour pouvoir la 
rendre consommable et atténuer les effets de ces deux inconvénients, il a fallu mettre au point une technique pour la détoxifier. Cette technique très ancienne a été décrite dès le $\mathrm{XII}^{\mathrm{e}}$ siècle par le célèbre agronome andalou $\mathrm{Ibn} \mathrm{Al}$ Awwam dans son traité de l'agriculture « Kitāb Al-Filāḥa » dont voici un extrait :

«.... Quand on veut employer la vesce, on procède de la manière suivante: on prend la graine, on la met dans l'eau douce, de façon qu'elle y soit entièrement plongée et même que l'eau s'élève au-dessus. On renouvelle cette eau les soirs et tous les matins. On pose ensuite cette graine, pour la torréfier, dans une chaudière large ou sur une plaque placée sur un feu doux, ayant soin de remuer sans interruption; elle se dépouille de son écorce. Alors on fait moudre la vesce (ainsi décortiquée); on la pétrit et on en fait une pate qu'on fait cuire. On ne doit point manger la vesce (seule), parce-que c'est un aliment nuisible; mais on la mêle (sa farine) avec la farine de lentille ou de froment lavé, puis on complète la panification, et le pain se mange avec les substances que nous avons indiquées ".

21 Les anciens types de consommation des graines de l'orobe correspondent donc à deux types d'usages : l'alimentation et la pharmacopée.

En ce qui concerne l'alimentation nous avons relevé deux principaux types d'usages (Berque 1978, Al Khattabi 1990, Nekadi 2007) : la préparation de bouillie ou soupe et du pain. Dans les deux cas, les grains doivent être traités avant de les moudre pour obtenir de la farine. En ce qui concerne les farines, il faut insister sur un point important dans l'histoire des graines utilisées. En effet, la différence entre céréales et légumineuses telle qu'elle est connue aujourd'hui n'a pas toujours existé et les légumineuses étaient souvent utilisées comme source de farine pour faire du pain. Différentes sources attestent de l'utilisation de la farine des légumineuses en général et de l'orobe en particulier pour la préparation des pains. À ce sujet, on peut citer Nekadi (2007), qui dans son ouvrage "L'agriculture en Andalousie pendant le siècle v de l'hégire » affirme que pour cette époque on ne faisait pas la distinction entre céréales et légumineuses pour la fabrication de pains et on utilisait la farine de lentilles, fèves, pois et orobe. Au Maroc et pour la même période, Abi Marwan Abdelmalek Ibn Zouhair Al Ayadi ${ }^{8}$ contemporain du calife almohade Abdel Moumen Ben Ali, parle du pain de «kersannah » comme un pain de mauvaise qualité dans son livre sur l'alimentation intitulé «Kitāb al-aghdiya ». De même, Berque (1978), cite l'utilisation des graines de Vicia ervilia, dans le haut atlas $d u \mathrm{Xv}^{\mathrm{e}}$ au $\mathrm{xIX}^{\mathrm{e}}$ siècle pour la production de pain. En ce qui concerne le deuxième type d'usage alimentaire, Berque (1978) cite l'utilisation de la farine de Vicia ervilia pour la préparation d'une bouillie appelée dans le Haut-Atlas en berbère « tagulla ». Rosenberger (2001), parle de l'ers ou vesce noire parmi les cultures complémentaires et les nourritures de substitution pendant les périodes de disettes du $\mathrm{XV}^{\mathrm{e}}$ au $\mathrm{XVIII} \mathrm{e}^{\mathrm{e}}$ siècle. Pour des périodes plus récentes, nos travaux sur le terrain avec la population locale des Jbala, nous ont permis de mentionner des usages analogues sous forme de purée au cours des dernières famines que la région a connues entre 1940-1945. Pendant la même période, une dame âgée de la tribu des Anjra nous a décrit

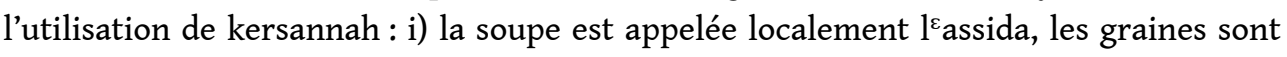
trempées complètement dans de l'eau pendant deux nuits, avec un changement de l'eau matin et soir ; ils sont placés ensuite dans une marmite à feu doux, en les remuant de temps en temps, jusqu'à homogénéisation ii) le pain: la farine de Vicia ervilia est bouillie dans de l'eau jusqu'à obtention d'une pâte bien cuite, les petites boules sont placées dans des plats pour les sécher, ce pain ou galette se mange avec du beurre salé (smen) ou de l'huile d'olive. 
23 En ce qui concerne les usages en tant que plante médicinale, son utilisation dans la pharmacopée marocaine traditionnelle a été décrite par Bellakhdar (1997). La farine d'orobe mélangée à de l'huile et du vinaigre serait utilisée pour faire des cataplasmes sur les morsures et les plaies. Elle est aussi utilisée en mélange avec l'huile d'olive contre la toux. Elle a été citée dans différentes travaux d'ethnopharmacologie portant sur la reconstitution des pratiques et du savoir médical au Moyen Âge en Égypte et en Jordanie (Lev \& Amar 2002 et 2008). Planchon (1869), la cite parmi les quatre farines résolutives ${ }^{9}$ dans son « Histoire naturelle des drogues simples ».

\section{L'orobe ou kersannah dans les traités d'agronomie et d'alimentation arabes}

Le livre d'Abou L'Khayr Al Ishbili " ${ }^{\mathrm{c} U m d a t ~ a l-t ̦ a b i ̄ b ~ f i ~ m a c r i f a t i ~ a l ~ n a b a t ~}{ }^{10} \mathrm{du}$ XII ${ }^{\mathrm{e}}$ siècle correspond à un catalogue botanique des plantes reconnues et nommées à cette époque. Il cite "kersannah »" qu'il présente comme le nom commun utilisé en Andalousie en faisant référence aux noms latins, grec, persan et syriaque. Il ne parle pas des usages ou utilisations de la plante, mais signale l'existence de deux formes qui se différencient essentiellement par la taille de la graine et de la plante. La forme à grande taille correspondrait à la forme cultivée et serait bien reconnue par les agriculteurs et la petite forme correspondrait à une forme spontanée. Cette description est conforme à l'état des connaissances actuelles ou on reconnaît, à côté des formes cultivées, des formes spontanées dans la nature et qui seraient des formes sauvages ou adventices.

Dans la traduction en français ${ }^{12}$ du livre de l'agriculture «Kitāb Al-Filāḥa » d'Ibn Al Awwam, (XII ${ }^{\mathrm{e}}$ siècle) on note l'absence de citation du nom arabe «kersannah " mais seulement les noms français vesce noir et ers. L'auteur lui consacre un article ${ }^{13}$ dans le chapitre dédié à la culture des légumineuses où il expose aussi bien les techniques agriculturales associées à cette culture que les utilisations et usages des graines.

$26 \mathrm{Au} \mathrm{XVI}{ }^{\mathrm{e}}$ siècle (1585), Abou Kassem Ben Mohamed Al Ghassani Al Wazir cite " kersannah » dans " hadīqat al-Azhar fīmahiyat al- ${ }^{c}$ ushb wa al-c uqqār » ${ }^{14}$. En ce qui concerne la présentation de la plante, il reprend à peu près la même description que celle d'Abou Al Khayr de Séville. Mais comme l'auteur est médecin, il a consacré un paragraphe aux usages en pharmacopée et a exposé les avantages et les inconvénients de son utilisation dans l'alimentation.

$\mathrm{Au} \mathrm{XVIII}^{\mathrm{e}}$ siècle on peut citer un manuscrit intitulé « Kāshef Er-Romūz » d'Abd Erezzaq Ed-Djezairy traduit et annoté par Leclerc en 1874. Il cite le nom arabe de « kersannah » et le traduit par orobe et il parle seulement de ses usages médicinaux.

\section{La culture de l'orobe au Maroc}

\section{Périodes précoloniale et coloniale}

Plusieurs sources mentionnent l'utilisation de l'orobe aux périodes précoloniales et coloniales.

Michel (1997), dans son ouvrage consacré au Maroc précolonial, cite «kersannah » (orobe) comme une culture qui caractérisait les collines pré-rifaines et le Rif. 
Dans son étude d'une tribu marocaine les Fahçya ${ }^{15}$, Salmon (1904) cite parmi les légumineuses cultivées «kersannah » en prenant soin de préciser qu'elle est utilisée surtout pour la nourriture des bœufs.

Le comte de La Revelière (1917), cite «kersannah » (orobe), parmi les légumineuses cultivées comme fourrage et utilisées dans l'alimentation des bœufs de labour.

Pour la zone du Rif sous protectorat français, Thoyer (1937) présente la culture de « kersannah » ou orobe comme une culture de la zone sud-rifaine et presque exclusive du territoire de Taza où elle couvre 6050 ha. L'orobe est généralement utilisée comme nourriture pour le bétail. Elle favoriserait la lactation des vaches laitières et augmenterait la résistance des bœufs de labour. Dans les années de mauvaises récoltes, elle remplace le blé et l'orge dans l'alimentation des paysans. Les cultivateurs connaissent sa toxicité et ils procèdent à la macération dans l'eau plusieurs jours avant de la décortiquer et la consommer. Les colons français la cultivaient rarement avec seulement 15 ha en 1936 pour l'utiliser dans l'alimentation du bétail. Mais ils s'en méfiaient car ils la considéraient comme toxique pour les porcs. D'ailleurs, Chapuis et al. (1937) ont relaté des cas d'intoxications par l'orobe chez des colons de la région de Fès et qui ont touché des vaches tarentaises. Velu (1937), relativise la dangerosité de l'orobe et explique ces accidents par la méconnaissance des usages et utilisations des colons contrairement aux cultivateurs locaux. En effet, les colons auraient utilisé l'orobe comme fourrage vert à un stade de développement où la plante est toxique. Cet état des choses n'est pas propre à l'orobe mais partagé avec d'autres plantes fourragères comme le sorgho et d'autres espèces de vesce. Au contraire, il a recommandé son utilisation pour l'engraissement des animaux en association avec d'autres aliments.

Dans la zone du Rif sous protectorat espagnol, vu la nature du relief et la prédominance des montagnes, les superficies agricoles sont beaucoup moins importantes que dans la zone sous protectorat français. Ils correspondent à seulement 8,6\% du territoire et n'excéderaient pas 168000 ha dans les estimations de 1936-1938 ${ }^{16}$. Pourtant, l'orobe ressort dans les statistiques comme une culture importante. En effet, c'est la troisième légumineuse cultivée après la fève et les pois chiches et avant les haricots et les lentilles (Figure 1). 
Figure 1 : Évolution de la production de l'orobe comparée à celle d'autres légumineuses alimentaires dans la zone nord du Maroc avant l'indépendance, entre 1936 et 1941

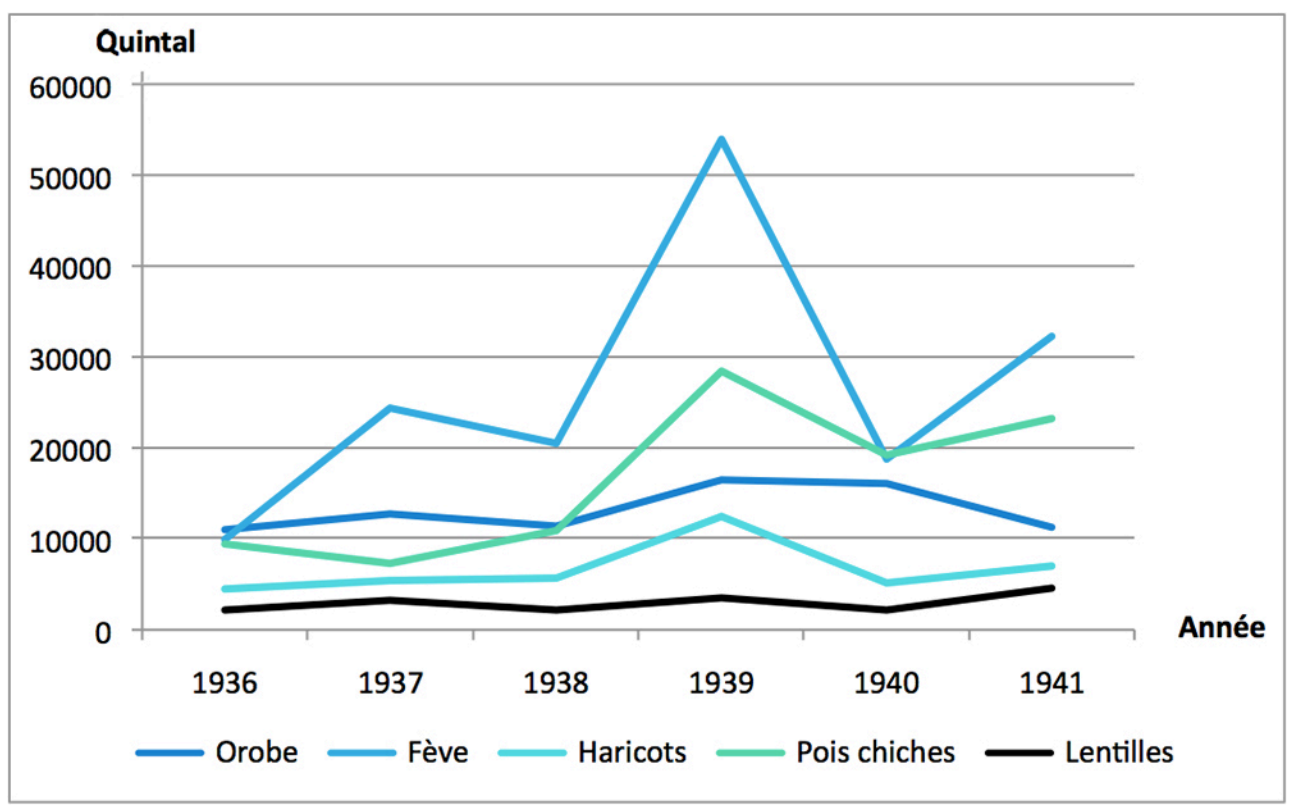

Réalisé à partir des données extraites de Ministerio de trabajo Dirección general de estadística 1942, Zona de protectorado y de los territorios de sobernia de España en el Norte de Africa, Anuario Estadístico. Madrid

\section{Situation actuelle de la culture de l'orobe}

Au Maroc, elle est encore cultivée dans le Rif (Francis et al. 1994, Enneking 1995), et dans les régions d'Aarbaoua, Ouezzane, Pré-Rif, Fès et Taza (Foury 1954, Hmimsa \& Ater 2008).

Selon les données disponibles ${ }^{17}$, l'importance de cette culture au Maroc est variable d'une année à l'autre (Figure 2). Par exemple, au cours de la dernière décennie, sa production a connu d'importantes fluctuations. 
Figure 2 : Évolution de la superficie emblavée et de la production de l'orobe au Maroc après l'indépendance, entre 1993 et 2007

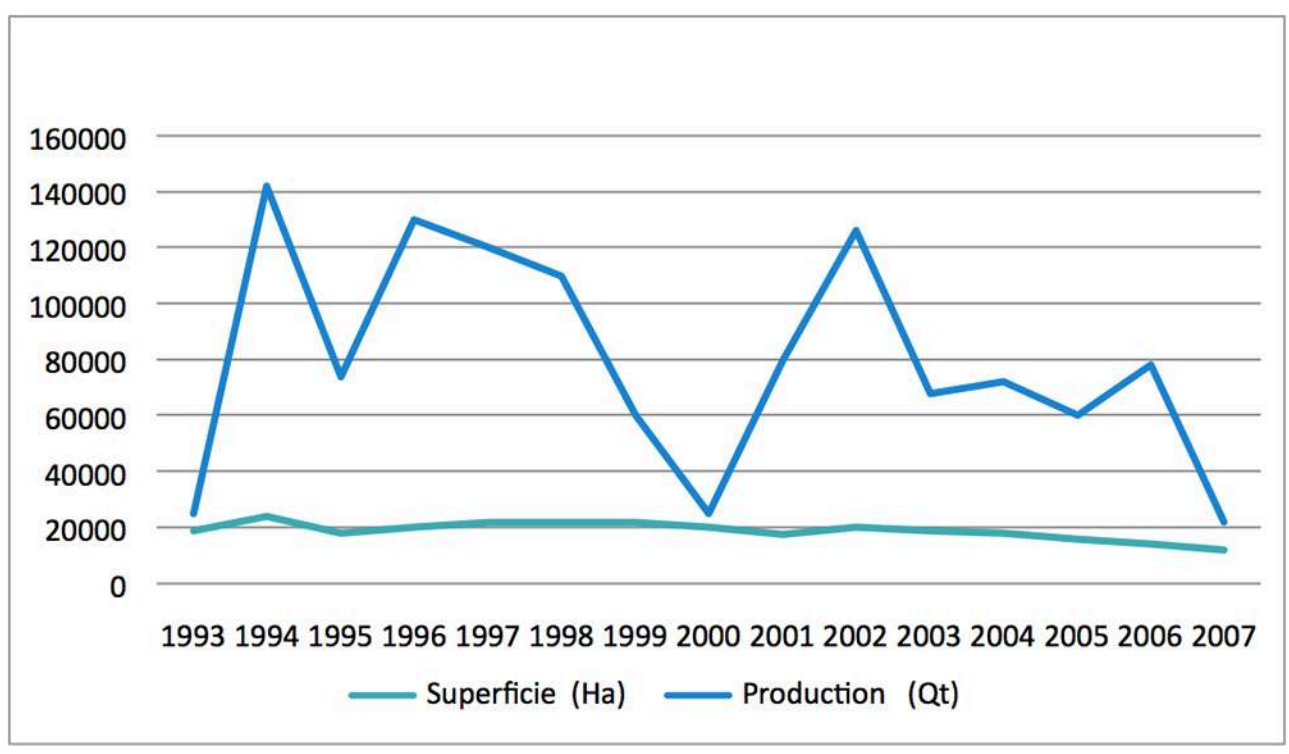

Réalisé à partir des données de la Direction des Statistiques, 2008. Ministère de l'Agriculture et de la Pêche maritime, Rabat

En effet, un maximum a été enregistré en 1994 où la superficie cultivée était de l'ordre de $23000 \mathrm{ha}$, avec une production de $142400000 \mathrm{~kg}$, contre seulement $25100000 \mathrm{~kg}$ au cours d'une autre saison. Cependant, d'une manière générale, la tendance est à la régression. Les fluctuations peuvent s'expliquer par les conditions climatiques défavorables de certaines saisons alors que la régression s'expliquerait par la marginalisation de cette culture.

$\mathrm{Au}$ cours de nos travaux sur l'agrodiversité dans les agroécosystèmes traditionnels des montagnes du Rif (Hmimsa \& Ater 2008) nous avons reconnu la présence de cette culture dans 65 dchars $^{18}$ sur 187 visités ce qui représente $35 \%$ des sites prospectés en 2006. Une prospection dans le cadre de nos travaux sur Vicia ervilia en 2009 a montré une forte diminution de la fréquence de cette culture (Figure 3). En effet, elle a été retrouvée seulement dans 19 dchars ce qui correspond à une différence d'environ $70 \%$ enregistrée en l'espace de trois années. Ces résultats confirment les risques de l'accélération de la régression de cette culture et de sa marginalisation. 
Figure 3 : Évolution de la culture de l'orobe dans le nord-ouest du Maroc à partir des enquêtes de terrains recensant les sites où la culture est encore pratiquée
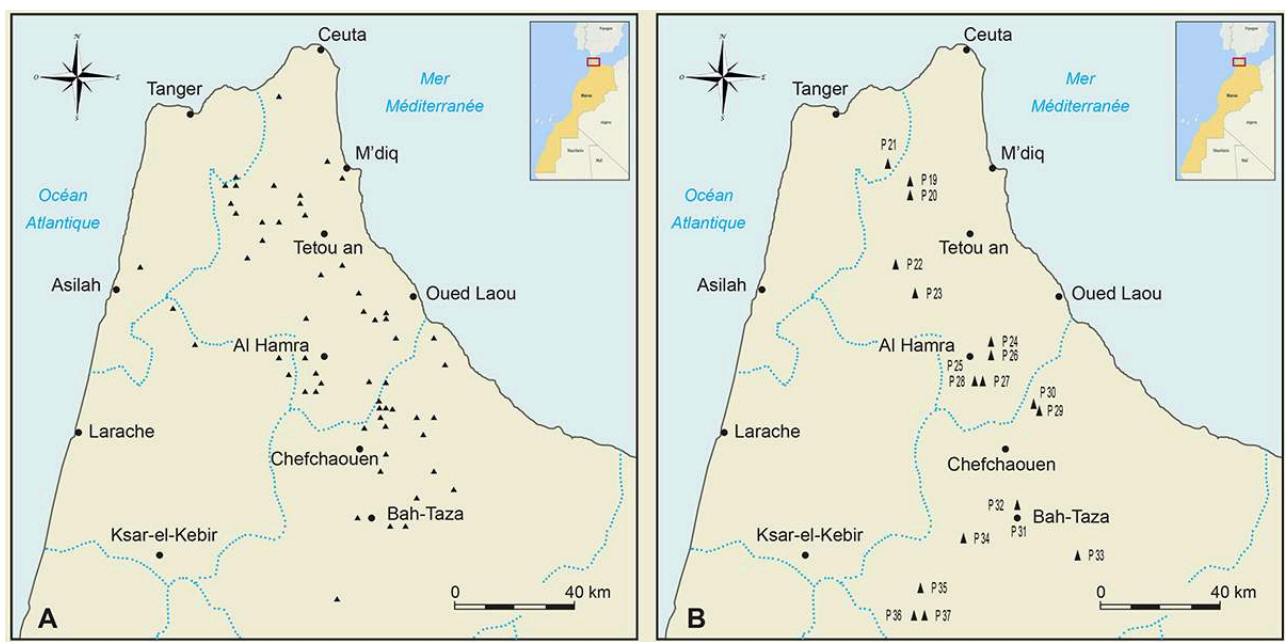

A. Situation en 2006. B. Situation en 2009

Carte élaborée par Y. Hmimsa avec Diva GIS version 7.5.0, free software disponible à http://www.divagis.org/

\section{Les pratiques culturales et usages dans les agroécosystèmes traditionnels du Rif}

La culture de Vicia ervilia, est pratiquée essentiellement sur des petites parcelles qui ne dépassent guère $300 \mathrm{~m}^{2}$. Les semences sont d'origine locale et appartiennent généralement au paysan ( $87,5 \%$ des cas recensés). Dans ce sens, un agriculteur peut garder son propre stock de semences pour une durée variable à condition de ne pas les battre et garder la plante sèche toute entière (Figure 4). Dans certains cas relativement limités, les semences peuvent être achetées dans les souks hebdomadaires (12,5\%). Le prix des graines est faible et fluctue entre un minimum pendant la période de récolte (5-6 Dh/ kg) et un maximum pendant la période des semis $(8-9 \mathrm{Dh} / \mathrm{Kg})$.

Figure 4 : Culture de l'orobe dans les agroécosystèmes traditionnels du nord du Maroc
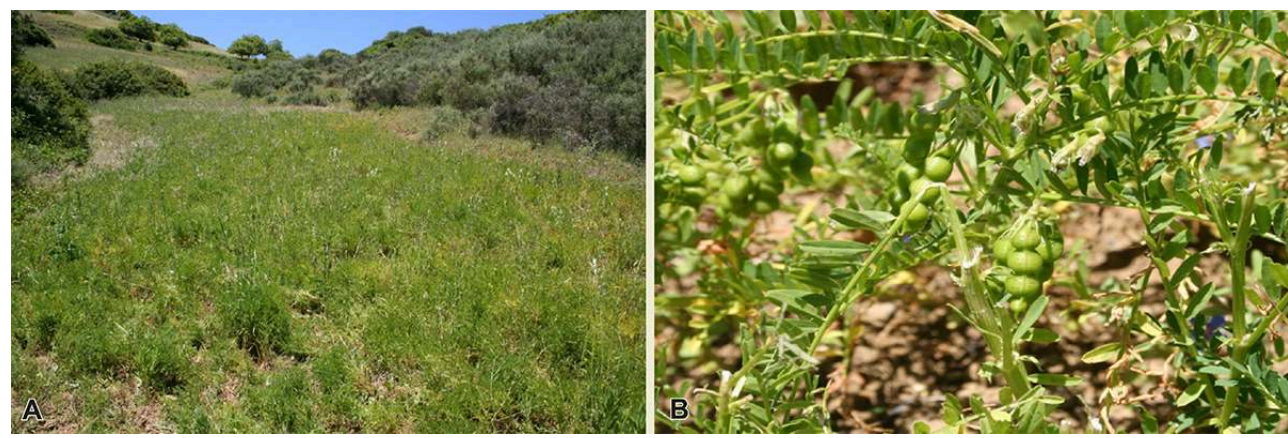

A. Vue d'une parcelle cultivée d'orobe. B. Plant d'orobe montrant une gousse avant maturation (C) S. El Fatehi et M. Ater

Vicia ervilia est utilisée exclusivement pour l'alimentation des animaux. Ses graines sont écrasées puis données en mélange avec d'autres graines telles que l'orge, l'avoine et le sorgho. En fait, dans la région, elle est destinée à l'alimentation des caprins et des 
bovins. Les paysans déclarent l'apprécier pour différents usages dans les pratiques d'élevage. Notamment pour l'engraissement $(76,47 \%)$, la production du lait $(35,3 \%)$ et aussi comme un stimulant pour les animaux de trait (17,65 \%).

Du point de vue calendrier des pratiques agricoles concernant la culture de Vicia ervilia, $77 \%$ des agriculteurs commencent le labour au mois d'octobre, alors que $23 \%$ déclarent démarrer plus tardivement cette activité (mois de janvier). Le semis se fait toujours à main levée. La durée de cette culture varie entre cinq et huit mois, car la récolte se fait $\mathrm{du}$ mois de mai à juin. Le battage se fait manuellement suivant la pratique traditionnelle (Figure 5). Après la récolte, le champ est utilisé pour le pâturage. Les champs destinés à la culture de Vicia ervilia ne réclament ni entretien ni préparation particulière du sol. Cependant, un bon rendement nécessiterait quand même une fertilisation qui est généralement organique.

Figure 5 : L'un des auteurs (S. El Fatehi) pendant la réalisation des enquêtes de terrain en train d'interroger des agriculteurs sur une aire de battage traditionnel des céréales

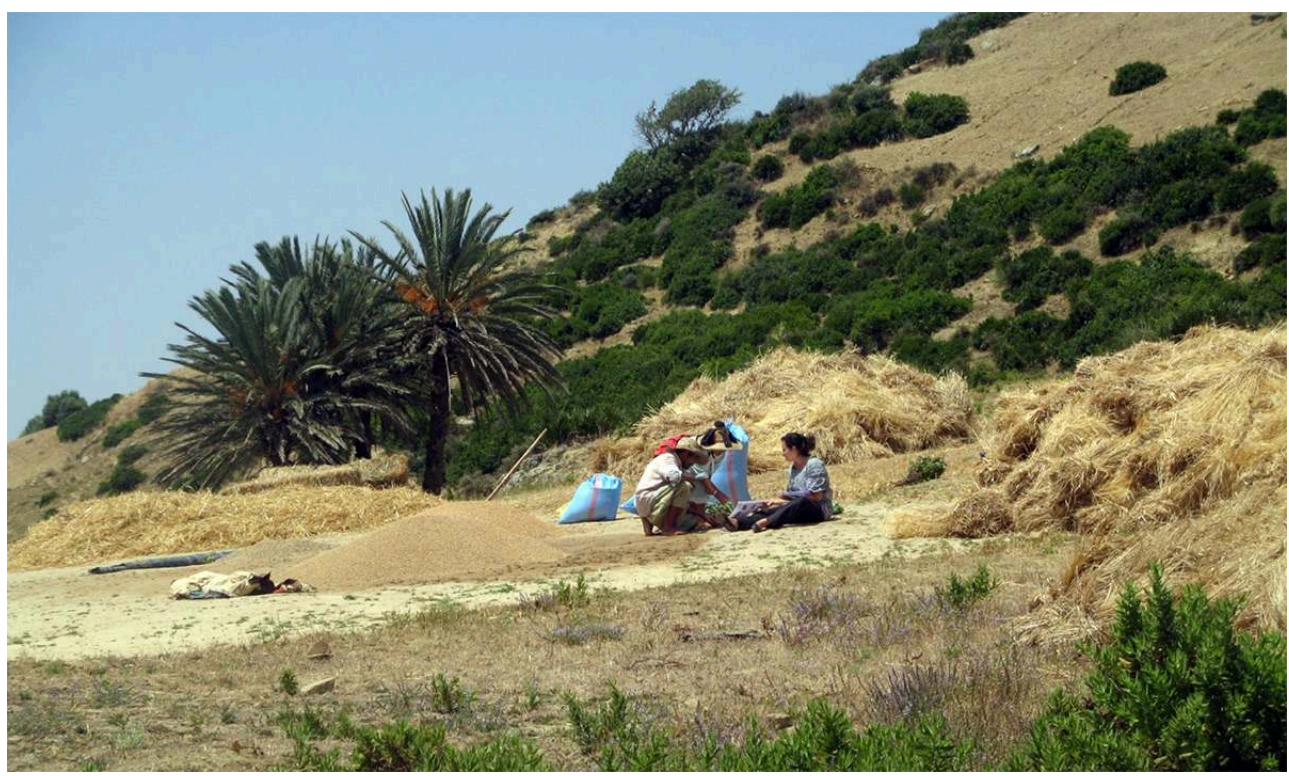

(c) M. Ater

\section{Conclusions}

La culture de l'orobe est très ancienne, car elle a existé depuis le début de la pratique de l'agriculture au Paléolithique en Méditerranée orientale. En Méditerranée occidentale et dans nos régions, les signes de cette culture sont plus récents mais ils datent quand même de la Protohistoire (Néolithique inférieur et âge de bronze). Il est donc évident que la pratique de cette culture existait dans nos régions bien avant l'Antiquité et ne serait pas liée à des introductions dans des périodes historiques plus récentes ${ }^{19}$. Il s'agit bien d'une culture autochtone ancienne.

C'était une culture importante et elle figurait parmi les légumineuses cultivées et utilisées en Andalousie et au Maghreb du Moyen Âge à l'époque moderne. En effet, différents traités arabes d'agronomie, d'alimentation et de médecines $d u \mathrm{XII}^{\mathrm{e}} \mathrm{au} \mathrm{XVII}^{\mathrm{e}}$ citent cette culture. Contrairement à la période actuelle, les utilisations et les usages de 
l'orobe en tant que légume sec étaient plus importants. Comme il était d'usage dans ces périodes, les graines des légumineuses servaient aussi pour produire de la farine. Bien qu'elle ne fût pas très appréciée, elle était utilisée dans l'alimentation humaine pour préparer du pain, des galettes ou des bouillies. Cette utilisation était particulièrement importante pendant les périodes de disette et à ce titre l'orobe fait partie des aliments de substitutions en réponse à la précarité. Actuellement, l'utilisation de l'orobe dans l'alimentation humaine a été abandonnée. Le seul usage encore reconnu de cette culture est l'alimentation animale en vert comme fourrage ou à partir des graines.

La culture de l'orobe a connu une marginalisation à l'image d'autres anciennes plantes cultivées. Cette marginalisation s'est accélérée le siècle dernier même si cette culture mineure a été conservée dans les zones d'agricultures traditionnelles du Rif. Dans le contexte actuel de la prise de conscience de l'importance de l'agrobiodiversité pour répondre aux nouveaux défis face aux changements globaux, la reconnaissance et la conservation des ressources génétiques de ce type est plus que d'actualité.

\section{BIBLIOGRAPHIE}

Al Khattabi M.L. 1995 - Umdat al-Ṭabỉb fi Ma'rifat al-Nabāt par Abū al-Khayr Al-Ishbīlī. Edition annotée (Guide des plantes à l'usage du médecin). Rabat, Ed. Dar Al Gharb Al Islami, 1024 p.

Al Khattabi M.L. 1985 - Hadīqat al-Azhar fï mahiyat al- ${ }^{c}$ ushb wa al-c ${ }^{c}$ qqā̄r, par Abou Al Kassem Ben Mohamed Al Ghassani Al Wazir. Edition annotée et introduite. Beyrut, Ed. Dar Al Gharb Al Islami, $427 \mathrm{p}$.

Al Khattabi M.L. 1990 - Kitab al aghdiya de Abi Marwan Abdelmalek Ibn Zouhair Al Ayadi. In : Pharmacopée et régimes alimentaires dans l'œuvre des auteurs hispano-musulman. Textes choisis et commentés. Beyrut, Ed. Dar Al Gharb Al Islami.

Ater M. \& Hmimsa Y. 2008 - Agriculture traditionnelle et agrodiversité dans le bassin versant de l'Oued Laou. Travaux de l'Institut Scientifique, série générale 5 : 107-115.

Ater M. \& Hmimsa Y. 2013 - Agrodiversité des agroécosystèmes traditionnels du pays Jbala (Rif, Maroc) et produits de terroirs. In : Ilbert H., Tekelioğlu Y., Çağatay S. \& Tozanli S. (Ed.) Indications Géographiques, dynamiques socio-économiques et patrimoine bio-culturel en Turquie et dans les pays méditerranéens ». Montpellier, CIHEAM (Centre International de Hautes Études Agronomiques Méditerranéennes), Série A : Séminaires Méditerranéens, $N^{\circ} 104$, Options Méditerranéennes : 197-208.

Ballouche A. \& Marinval P. 2003 - Données palynologiques et carpologiques sur la domestication des plantes et l'agriculture dans le néolithique ancien du Maroc septentrional (Site de Kaf That el-ghar). Archéométrie $27: 49-54$.

Barkai R. 1998 - A history of jewishgyna ecological texts in the middle ages. Leiden, New York, Köln, Ed. Brill, $241 \mathrm{p}$.

Bellakhdar J. 1997 - La pharmacopée marocaine traditionnelle. Casablanca, Ed. Le Fennec, Paris, Ibis, $764 \mathrm{p}$. 
Berque J. 1978 - L'intérieur du Maghreb XV'e-XIXe siècle. Paris, Gallimard, 546 p.

Bouby L. 2003 - De la récolte au stockage : éclairages carpologiques sur les opérations de traitement des céréales à l'âge du Bronze dans le sud de la France. In : Anderson P.C., Cummings L.S., Schippers T.K. \& Simonel B. (Ed.), Le traitement des récoltes: un regard sur la diversité, $d u$ Néolithique au présent. XXIII ${ }^{\mathrm{e}}$ rencontres internationales d'archéologie et d'histoire d'Antibes. Antibes, Éditions APDCA : 21-46.

Bounejmate M. 1997 - Le patrimoine fourrager et pastoral au Maroc : acquis et perspectives. In : Birouk A. \& Rejdali M. (Ed.), Ressources phytogénétiques et développement durable. Rabat, Actes Editions : 219-238.

Buxo R. 1997 - Arqueología de las plantas. La explotación económica de las semillas y los frutos en el marco mediterráneo de la Península Ibérica. Barcelone, Critica, 368 p.

Carnoy A. 1958 - Noms grecs de plantes d'origine pré-hellénique. L'antiquité classique 7 (2) : 305-327.

Castañeda-Álvarez N.P., Khoury C.K., Achicanoy H.A., Bernau V., Dempewolf H., Eastwood R.J., Guarino L., Harker R.H., Andy Jarvis A., Maxted N., Müller J.V., Ramirez-Villegas J., Sosa C.C., Struik P.C., Vincent H. \& Toll J. 2016 - Global conservation priorities for cropwild relatives. Nature plants Article number: 16022 | Doi: 10.1038/NPLANTS. 2016. 22 p.

CBTHA 2002 - Conservation de la biodiversité par la transhumance dans le versant sud du haut atlas. Nomadic Peoples 6 (1) : 209-212.

Chapuis G., Girard \& Saillard 1937 - Intoxication par ingestion de kersannah. La Terre Marocaine $91: 15-16$.

Corriente F. 2008 - Dictionary of Arabic and allied loanwords Spanish, Portuguese, catalan, galician and kindred dialects. Leiden, Boston, DC publishers, $696 \mathrm{p}$.

El Fatehi S., Béna G., Filali-Maltouf A. \& Ater M. 2014 - Variation in yield component, phenology and morphological traits among Moroccan bitter vetch landraces Vicia ervilia (L.) Willd. African Journal of Agricultural Research 9 (23) : 1801-1809.

El Fatehi S., Béna G., Filali-Maltouf A. \& Ater M. 2016 - Genetic diversity of Moroccan bitter vetch Vicia ervilia (L.) Willd. landraces revealed by morphological and SSR markers. Australian Journal of Crop Science 10 (5) : 717-725.

Enneking D. 1994 - The biological chemistry of Vicia toxins. Ph D. thesis, University of Adelaide, South Australia.

Enneking D. 1995 - The toxicity of Vicia species and their utilization as grain legumes. Nedland, Centre for Legumes in Mediterranean Agriculture (CLIMA), 134 p. (Occasional Publication ; 6).

Enneking D. \& Miller N.F. 2014 - Bitter vetch Vicia ervilia: ancient medicinal crop and farmers favorite for feeding livestock. In : Minnis P.E. (Ed.) New lives of ancient and extinct crops : 254-268

Foury A. 1954 - Les légumineuses fourragères au Maroc (deuxième partie). Les Cahiers De La Recherche Agronomique, INRA, 5 : 287-658.

Francis C.M., Bounejmate M. \& Robertson L.D. 1994 - Observations on the distribution and ecology of Vicia and Lathyrus species in Morocco. Al Awamia 84: 17-42.

Gonzalez C.G. 2011 - Changement climatique, sécurité alimentaire et agrobiodiversité : vers un système alimentaire juste, résilient et durable. Fordham Environmental Law Review, Vol. 22, 493 p. 
Hernández Bermejo J.E. \& León J. 1994 - Cultures marginalisées 1492 : une autre perspective. Rome, FAO, 354 p. (Production végétale et protection des plantes ; 26).

Hillman G.C. 1975 - The plant remains from Tell Abu Hureyra: a preliminary report. The excavation of Tell Abu Hureyra in Syria: a preliminary report. AMT Proceedings of the Prehistoric Society 41 : 70-73.

Hillman G.C., Colledge S.M. \& Harris D.R. 1989 - Plant-food economy during the Epipalaeolithic period at Tell Abu Hureyra, Syria: dietary diversity, seasonality, and modes of exploitation. In : Harris D.R. \& Hillman G.C. (Ed.) Foraging and Farming. London, Unwin Hyman : 240-268.

Hillman G.C., Hedges R., Moore A., Colledge S. \& Pettitt P. 2001 - New evidence of Lateglacial cereal cultivation at Abu Hureyra on the Euphrates. The Holocene. 11 (4) : 383-393.

Hmimsa Y. \& Ater M. 2008 - Agrodiversity in the traditional agrosystems of the Rif mountains (north of Morocco). Biodiversity: Journal of life on earth 9 (1 \& 2) : 78-81.

Ibn Al Awwam 2000 - Kitāb Al-Filāha (Le livre de l'agriculture). Edition revue et corrigée avec introduction de El Faïz. Actes Sud et Sinbad, 1052 p.

ILDIS World Database of Legumes 2010 - http://ww2.bgbm.org/EuroPlusMed/PTaxonDetail.asp? NameCache=Vicia\%20ervilia\&PTRefFk=8500000 (Consulté le 19/02/2012)

La Revelière 1917 - Les énergies françaises au Maroc. Paris, Plon, 561 p. (Études économiques et sociales).

Leclerc L. 1874 - Kachef er-roumouz (Révélations des énigmes) d'Abd Erezzaq Ed-Djezairy ou traité de matière médicale arabe d'Abd Erezzaq Ed-Djezairy. Paris, 411 p.

Lev E. \& Amar Z. 2002 - Ethnopharmacology survey of traditional drugs sold in the Kingdom of Jordan. Journal of Ethnopharmacoly $82: 131-145$.

Lev E. \& Amar Z. 2008 - Fossils of practical medical knowledge from medieval Cairo. Journal of Ethnopharmacology $119: 24-40$.

Lev E., Kislev M. \& Bar-Yosef O. 2005 - Mousterial vegetal food in Keba Cave. Mt. Carmel. Journal of Archaeological Science 32 : 475-484.

Michel N. 1997 - Une économie de subsistances : le Maroc précolonial. Le Caire, Institut Français d'Archéologie Orientale (IFAO), 2 vol.

Miège J.L. 1938 - Les cultures complémentaires au Maroc. Direction des affaires économiques. Rabat, Centre de recherche agronomiques. $339 \mathrm{p}$.

Ministerio de trabajo Dirección general de estadística 1942 - Zona de protectorado y de los territorios de sobernia de España en el Norte de Africa. Madrid, Anuario Estadístico.

Montemayor J. 1996 - Tolède entre fortune et déclin (1530-1640). Presses universitaires de Limoges, $521 \mathrm{p}$.

Morales J., Pérez-Jordà G., Peña-Chocarro L., Zapata L., Ruíz-Alonso M., López-Sáez J.A. \& Linstädter J. 2013 - The origins of agriculture in North-West Africa: macro-botanical remains from Epipalaeolithic and Early Neolithic levels of Ifri Oudadane (Morocco). Journal of Archaeological Science 40 : 2659-2669.

Nekadi Y. 2007 - Azzeraa fi Al Andalous khilala al karn al khamis hegri (L'agriculture en Andalousie pendant le siècle $v$ de l'Hégire). Oujda, Ed Al Jossour.

Peña-Chocarro L. \& Zapata L. 2010 - Neolithic agriculture in southwestern Mediterranean region. In : Gibaja J.F. \& Faustino Carvalho A. (Ed.) Os últimos caçadores-recolectores e as primeiras 
comunidades produtoras do sul da Península Ibéricae do norte de Marrocos. Universidade do Algarve, Algarve: 191-198. (Promontoria Monográfica ; 15).

Pérez-Jordà G., Peña-Chocarro L. \& Morales J. 2011 - Agricultura Neolítica en Andalucía: semillas y frutos. Revista de Prehistoria de Andalucía 2 : 57-72.

Perreault T. 2005 - Why chacras (swidden gardens) persist: Agrobiodiversity, food security, and cultural identity in the Ecuadorian Amazon. Human Organization 64 (4): 327-39.

Planchon G. 1869 - Histoire des drogues simples ou cours d'histoire naturelle. Sixième édition corrigée et augmentée. Paris, J. B. Baillière et Fils, 583 p.

Rosenberger B. 2001 - Société, pouvoir et alimentation. Nourriture et précarité du Maroc précolonial. Rabat-Riyad, Alizés, 315 p.

Salmon G. 1904 - Une tribu marocaine : les Fahçya. Archives marocaines. Publication de la mission scientifique du Maroc. Tome premier. Paris, Ernest le Roux : 149-261.

Thoyer J. 1937 - L'orobe. La Terre Marocaine 90 : 17-18.

Van Zeist W. \& Bakker-Heers J.A.H. 1986 - Archaeobotanical studies in the Levant 2-Neolithic and Halaf levels at Ras Sharma. Palaeohistoria $26: 151-170$.

Van Zeist W. \& Casparie W. 1968 - Wild einkorn wheat and barley from Tell Mureybet in northern Syria. Acta Botanica Neer landica 17 : 44-53.

Velu H.1937 - Notes additionnelles sur le Kersannah 91 : 16-17.

Villax E.J. 1963 - La culture des plantes fourragères méditerranéenne. Cahiers de la recherche Agronomique 17 : 506-508.

Zapata L., Peña-Chocarro L., Pérez-Jordà G. \& Stika H.P. 2004 - Early Neolithic agriculture in the Iberian Peninsula. Journal of World Prehistory 18 (4) : 283-325.

Zimmerer K.S., Córdova-Aguilar H., Olmo R.M., Olivencia Y.J. \& Vanek S.J. 2016 - Mountain Ecology, Remoteness, and the Rise of Agrobiodiversity: Tracing the Geographic Spaces of Human - Environment Knowledge. Annals of the American Association of Geographers 107 (2) : 441-4555

Zimmerer K.S. \& De Hann S. 2017 - Agrobiodiversity and a sustainable food future. Nature Plants 3 : 17047 | Doi: $10.1038 /$ nplants 47 p.

Zohary D., Hopf M. \& Weiss E. 2012 - Domestication of plants in the Old World. New York, Oxford University Press. $3^{\mathrm{e} e ́ d}$., 264 p.

\section{NOTES}

1. Système Ingénieux du Patrimoine Agricole Mondial, lancé par la FAO (2002) dans le cadre du Sommet mondial du développement durable à Johannesburg (Afrique du Sud).

2. Suivant les règles de la nomenclature botanique, le nom scientifique de l'espèce est un binôme en latin suivi des initiales du nom de l'auteur et de l'année de la publication.

3. Le premier nom scientifique accordé à un taxon.

4. Alabeja, Alarceña, Albejanca loca, Alberja, Alberjas, Alcaraceña, Alcarceña, Alcarcena de Toledo, Alcarceño, Alcaruna, Alcaruña, Alverja, Arabeja, Arveja, Chícharo, Chicharro, Erbellos, Ervilla, Giron, Girón, Herén, Hiero, Jirón, Labeja, Lenteja bastarda, Panujo, Pitos,Seros, Tito, Titos, Yedro, Yedros, Yerbo, Yero, Yero rubio, Yeros, Yeros blancos, Yerro et Yervo (ILDIS World Database of Legumes 2010). 
5. Ervilha de pombo, Ervilhaca-de-pombo, Ervilha-de-pombo, Gero, Gêro, Marroiço, Marroio, Marruiço, Orobo, órobo, Orobo bastardo, Orobo das boticas et Órobo-das-boticas.

6. Dans notre texte nous avons adopté « kerssanah » qui figure dans les textes agronomiques de la période du protectorat.

7. À l'origine, ces statistiques étaient obtenues à partir des relevés d'un impôt traditionnel le «tertib».

8. Le livre « Kitāb al-aghdiya »

9. Se dit d'une substance qui détermine la résolution d'un engorgement et qui calme une inflammation.

10. La traduction du titre arabe est « Guide des plantes à l'usage du médecin »; ouvrage annoté et présenté par Mohamed Al Arbi Al Khattabi et édité 1995.

11. Plante $n^{\circ} 11931: 320$.

12. La traduction de l'arabe a été faite par J-J. Clément-Mullet en 1887, nous avons consulté la version éditée en 2000 par Actes Sud.

13. Chapitre XXI, article $4: 588$.

14. Ouvrage annoté et édité par Mohamed Al Arbi Al Khattabi en 1985.

15. Texte faisant partie de la publication de la mission scientifique au Maroc de Salmon et Besnier, Archives Marocaines édition de 1904.

16. Zona de protectorado y de los territorios de soberanía de España en el Norte de Africa, Ministerio de trabajo Dirección general de estadística, Anuario Estadístico 1942. Madrid 1943.

17. Direction des Statistiques, Ministère de l'Agriculture et de la Pêche maritime 2008.

18. Correspond à une unité d'habitat rural dans le Rif et qui serait équivalent à un « hameau » en français.

19. Phéniciens, Carthaginois et Romains par exemple.

\section{RÉSUMÉS}

Dans cette contribution nous allons présenter certains aspects de l'histoire de la nomenclature et des usages de la culture de l'orobe (Vicia ervilia L. Willd.). Bien que cette culture soit actuellement considérée comme mineure et utilisée uniquement dans l'alimentation animale, cela n'a pas été toujours le cas. A certaines époques la culture de l'orobe était plus importante et occupait une place importante parmi les légumineuses cultivées. Même si elle était peu appréciée, elle était utilisée dans l'alimentation humaine et faisait partie de la pharmacopée traditionnelle. La marginalisation excessive de la culture et de la consommation de l'orobe est relativement récente. L'intérêt de la conservation de ce type de ressources est discuté à la lumière des recherches sur la durabilité et la résilience des agroécosystèmes face aux changements globaux.

In this contribution, we will present some aspects of the history, nomenclature and uses of the culture of the bitter vetch (Vicia ervilia L. Willd.). Although this crop is currently considered to be minor and used only in animal feed, this has not always been the case. At certain times in history, the cultivation of the bitter vetch was more important and had a significant place among the cultivated legumes. Although it was little appreciated, it was used in human food and was part of the traditional pharmacopoeia. The excessive marginalization of the culture and consumption of the bitter vetch is relatively recent. The value of conservation of this type of resource is 
discussed in the light of the search for sustainability and resilience of the agroecosystems in the face of global changes.

\section{INDEX}

Keywords : Vicia ervilia, ers, bitter vetch, kerssanah, nomenclature, history, uses, agroecosystems

Mots-clés : Vicia ervilia, ers, orobe, kerssanah, nomenclature, histoire, usages, agroécosystèmes

\section{AUTEURS}

\section{SALAMA EL FATEHI}

Enseignant chercheur à l'Université Abdelmalek Essaâdi, Département des Sciences de la Vie, Généticienne et ethnobotaniste, Membre du Laboratoire de Botanique Appliquée (Equipe BioAgrodiversité), Faculté Polydisciplinaire de Larache. B.P. 745, Poste Principale, Larache 92004 MAROC.

elfatehisalama@gmail.com

\section{MOHAMMED ATER}

Professeur de l'enseignement supérieur à l'Université Abdelmalek Essaâdi, Département de Biologie (Biologiste et Écologue, Membre du Laboratoire de Botanique Appliquée (Équipe BioAgrodiversité)), Faculté des Sciences, PB. 2062, Tétouan, 93030, MAROC.

mohammed.ater@gmail.com 